\title{
Design and Real Life Deployment of a Pervasive Monitoring System for Dementia Patients
}

\author{
Friedrich Hanser \\ Research Division for \\ Pervasive Health \\ UMIT, Hall i. Tirol,
}

Austria

Email: friedrich.hanser@umit.at

\author{
Agnes Gruenerbl \\ Research Division for \\ Pervasive Health \\ UMIT, Hall i. Tirol, \\ Austria
}

\author{
Clemens Rodegast \\ Embedded Systems Lab \\ University of Passau, \\ Germany
}

\author{
Paul Lukowicz \\ Embedded Systems Lab \\ University of Passau, \\ Germany
}

\begin{abstract}
We present the first phase of a project for monitoring elderly people suffering from dementia in the 'real life' environment of an 'assisted living' flat associated with a nursing home. Based on monitoring requirements derived in discussions with the nursing staff the system consists of a UBISENSE location system for monitoring location, a sound-system for monitoring vocal interaction, and the TECO 'Particles' sensor nodes mounted under the beds for sleep monitoring. In this paper we introduce the background and environment of the project, describe the requirements and the system and present some initial data collected in the first deployment period.
\end{abstract}

\section{INTRODUCTION}

So-called Ambient Assisted Living systems that use pervasive computing technology to assist the elderly and the handicapped have recently developed into a major application area. Technologies such as indoor location [1], activity monitoring [2], and wearable systems [8] are increasingly being presented and tested in the context of such applications. In [6] a survey of using new technology to support elderly people is presented and in [7] research for developing systems to enhance the quality of life of Alzheimer patients is described . Many 'smart' flats or houses as the 'Gator Tech Smart House' [3] or the 'Georgia Tech Aware Home' [4] have also been implemented, in which such systems can be tested under lab condition. However so far there has been only little work done in deploying complex pervasive computing systems in real-life conditions such as nursing homes, assisted living communities or elderly homes. This paper presents an ongoing project aimed at such a real-life deployment. We describe a system designed to monitor changes in the state of elderly people with dementia that has recently been deployed in an 'assisted living' community of the St. Katharina nursing home in Vienna, Austria. The system is based on the combination of location, vocal interaction, and sleep monitoring. The ultimate goal of our work is to determine how much information about the progress of dementia in the individual participants can be derived from the system. In this paper we describe the first phase of the project which has focused on system design, deployment and initial testing.

\section{A. The Project}

The work described in this paper is part of the 'Competence Center Light' Project (www.k-licht.at/index2.php) which aims to enhance the quality of life of elderly people suffering from dementia through an elaborate, optimally adapted indoor light system. A key issue in the project is the evaluation of the influence of the enhanced lighting on patients in different stages of dementia. A 1.5 year experiment in an assisted living flat of the St. Katharina nursing home in Vienna (www.bhs.or.at) has been devised for this purpose. The flat houses 13 inhabitants in different stages of dementia. The project uses a two track analysis of the inhabitants state.

First, psychologists will observe the activities of the inhabitants and conduct regular interviews (including interviews with the nursing staff). In parallel to the psychological monitoring a sensor system has been installed that will attempt to automatically detect the changes in the state of the patients. This sensor system is the main task of our group and the topic of this paper. The fact that sensor based monitoring will take place in parallel with professional psychological assessment over a long period of time is a unique chance for a solid, statistically significant validation of the system under real life conditions. Such a long term validation is the long term goal of the project.

\section{THE SYSTEM}

Assessing the state of a person suffering from dementia is not an easy task. Nursing staff often relies on personal impression and subtle non-verbal cues. For the sensor based evaluation the nursing and psychological staff have agreed to focus on three main criteria (1) the amount of time spent in the social areas, (2) the amount of social (in particular vocal) interaction and (3) the quality of sleep. Taking into account the fact that the monitoring system can not require the patients to carry elaborate sensor systems at well defined body locations a system consisting of the following components has been devised:

- UBISENSE Indoor Location System: The Ubisense 'Realtime location system' (www.ubisense.net) contains an array (at least two) of ultra-wide-band (UWB) radio 
receivers which calculate the location of UWB-signal transmitters (Tags). The system has been chosen because it provides location accurately enough to determine interaction patterns between people while at the same relying on a small Tag (about $8 \times 4 \times 1 \mathrm{~cm}$ ) that can be carried anywhere on the body.

- Microphones - location by sound: In order to determine the level of interaction between different persons, sound is recorded using commercially available microphones. We use beamforming [5] aligned with the UBISENSE signals in combination with spectral analysis to isolate individual persons speaking and thus get an estimate of the amount of vocal interaction. The basic concept of the beamforming algorithm is quite simple. The signals $x_{i}\left(t-\tau_{i}\right)$ captured by $i$ microphones are time shifted by $\tau_{i}$ samples. $\tau_{i}$ depends on the time difference of arrival between the sensors. The corrected signals are then summed up to receive the output signal $y(k)$.

$$
y(k)=\sum_{i} w_{i}(k) x\left(k-\tau_{i}\right)
$$

- Bed mounted accelerometers: An accelerometer based on the TECO Particle wireless sensor node (www.teco.edu/index 2.html/) has been mounted under each bed to assess sleep quality. The particle node also contains a light sensor that is used to detect when light is switched on/off and the curtains drawn.

Figure 1 shows a system overview as it has been deployed in the flat.
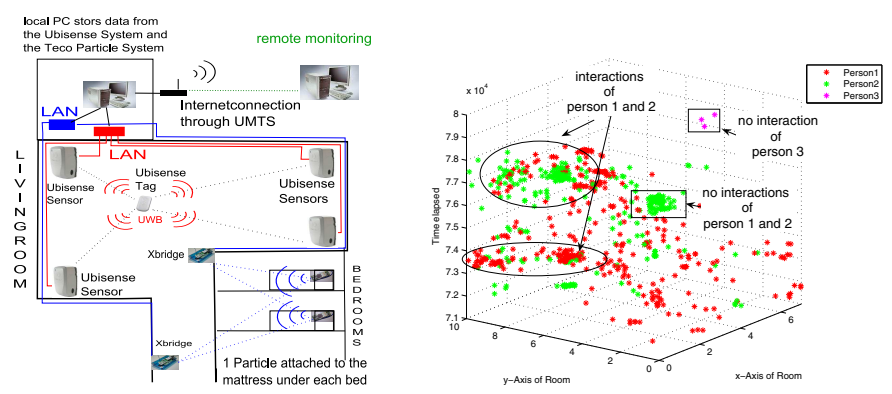

Fig. 1. Main configuration of the location system and the particles;
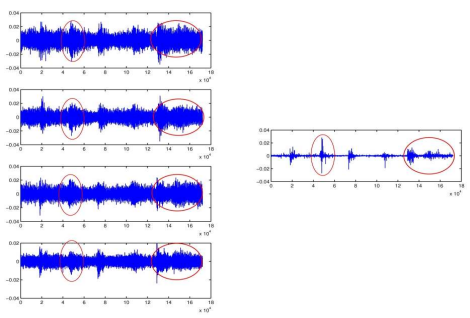

Fig. 2. Locations and interaction of 3 people in coherence to time;

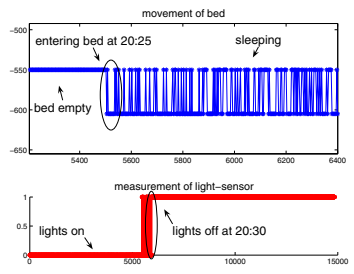

Fig. 4. Going to sleep and Fig. 3. Example results of beamforming turning off the light, measured to collect audio data from a known location by the 'Particle' sensors under of a person (see text for explanation).

\section{INITIAL DATA EXAMPLES}

The system has been deployed between August and September 2007 so that an evaluation of the correlation between sensor data and the gravity and development of the dementia in the inhabitants has not yet been possible. However, some initial data sets were collected

The analysis of an initial dataset recorded by the UBISENSE system at the nursing home is displayed in figure 2. It shows three persons staying in the monitored room, where two of them are staying for a longer period and are constantly moving around and partly interacting with each other, while the third person only stays for short at the same location without interacting with the others.

Initial experiments with beamforming are shown in Figure 4. The left part of the figure shows signals from each of the four microphones (each mounted in one corner of the room). The signal contains a considerable amount of noise (music playing in the room) and several instances of a person speaking from a well defined location (see red ellipses in figure 4). Right side shows the output of the system when it has been focused on this location using beamforming. It can be seen that the speech signal is reasonably well isolated.

Final example of data collected at the St. Katharina site is given in Figure 3 for the acceleration and light data from the particle nodes mounted under the bed. The time when the person was in the bed can be clearly recognized, and even small motions during sleep are visible. As affirmation to the acceleration data, figure 3 furthermore displays the data measured by the light sensor, which shows that the light is turned off a short time after entering bed.

\section{REFERENCES}

[1] L. M. Ni, Y. Liu, Y. C. Lau, and A. P. Patil. Landmarc: indoor location sensing using active RFID. Wirel. Netw., 10(6):701710, 2004.

[2] P.R. Liu, M.Q. Meng, P.X Liu, F.F. Tong, X.J.Chen. A telemedicine system for remote health and activity monitoring for the elderly. Telemed J E Health, 12(6):622-31, 2007.

[3] S. Helal, W. C. Mann, H. El-Zabadani, J. King, Yo. Kaddoura and E. Jansen. The Gator Tech Smart House: A Programmable Pervasive Space. IEEE Computer, 38(3):5060, 2005.

[4] G. Abowd, A. Bobick, I. Essa, E. Mynatt, and W. Rogers. The Aware Home: Developing Technologies for Successful Aging. In Proceedings of AAAI Workshop and Automation as a Care Giver. American Association of Artificial Intelligence (AAAI) Conference, Canada, 2002.

[5] L.J. Griffith, C. W Charles. An Alternative Approach to Linearly Constrained Adaptive Beamforming. IEEE Transactions on antennas and transactions, 301: 27-34, 1982.

[6] M.E. Pollack. Intelligent technology for an aging population: The use of AI to assist elders with cognitive impairment. AI Magazine, 26(2):9-24, 2005.

[7] H. Kautz, L. Arnstein, G. Borriello, O. Etzioni, and D. Fox. An overview of the assisted cognition project. Proceedings of the AAAI Workshop on Automation as Caregiver: The Role of Intelligent Technology in Elder Care, pages 60-65, 2002.

[8] E. Munguia Tapia, S. S. Intille, and K. Larson. Portable wireless sensors for object usage sensing in the home: Challenges and practicalities. In Proceedings of the European Ambient Intelligence Conference 2007, 2007 , p. to appear 